\title{
Fit Visualization and Simulation on Individual 3D Scanatars
}

\author{
Ulrich BOTZENHARDT \\ Human Solutions $\mathrm{GmbH}$, Kaiserslautern, Germany
}

\begin{abstract}
Individualization is one of the megatrends in the 21st century. This individualization contains not only changes in some areas of personal living - it is related to the whole life. Examples of individualization can be not only the new approach to the family division but also the ,joyful living" that stretches itself through the whole spectrum of today's life beginning with the individual creation of breakfast muesli, individually suited cosmetics, personally customizable sport shoes to personal trainer. In the apparel industry there are some innovative approaches and technologies like the Virtual Mirror available. The Virtual Mirror connects products and customers virtually in real time and gives the possibility to visualize products before they are produced. In a seamless process chain, body scanning is interactively connected with 3D clothing simulation and CAD software.
\end{abstract}

Keywords: megatrend, individualization, virtual mirror, avatar, scanatar, body scan, 3D, simulation, visualization, apparel

\section{Introduction and existing solutions}

This document describes the nowadays technologies in the apparel industry focusing on the latest developments of fit visualization and the benefits for the manufacturer of garments as well as for the end-customer.

There are various solutions and suppliers available. On one hand the solutions differ in its field of application beginning with fitting only up to made to measure of such complex garments as men suit, on the other hand there exists a variation of different types of tools and forms of distribution. There is a huge bandwidth available: paper order form or PDF, manufacturers' own B2B systems, online shops with manual measurement, home scanning with webcam or Kinect sensors, stationary 3D body scanner. Every solution has its own focus like web shop, scanning technology, etc..

\section{Solution}

\subsection{Overview}

INTAILOR links producers of tailored apparel collections with their own shops. It accompanies and supports the sales and production of individualized apparel or mass customization, from collection development right through to the point of sale and manufacturing - and all in one seamless system. Process chain is as follows:

- collection customer data

- generation customer's scan and transformation to scanatar

- selection of product and define the design and fitting definition

- simulation of the configured product

- interactive modification and simulation in design and fitting

- sending order and product manufacturing

\footnotetext{
*ulrich.botzenhardt@human-solutions.com
} 

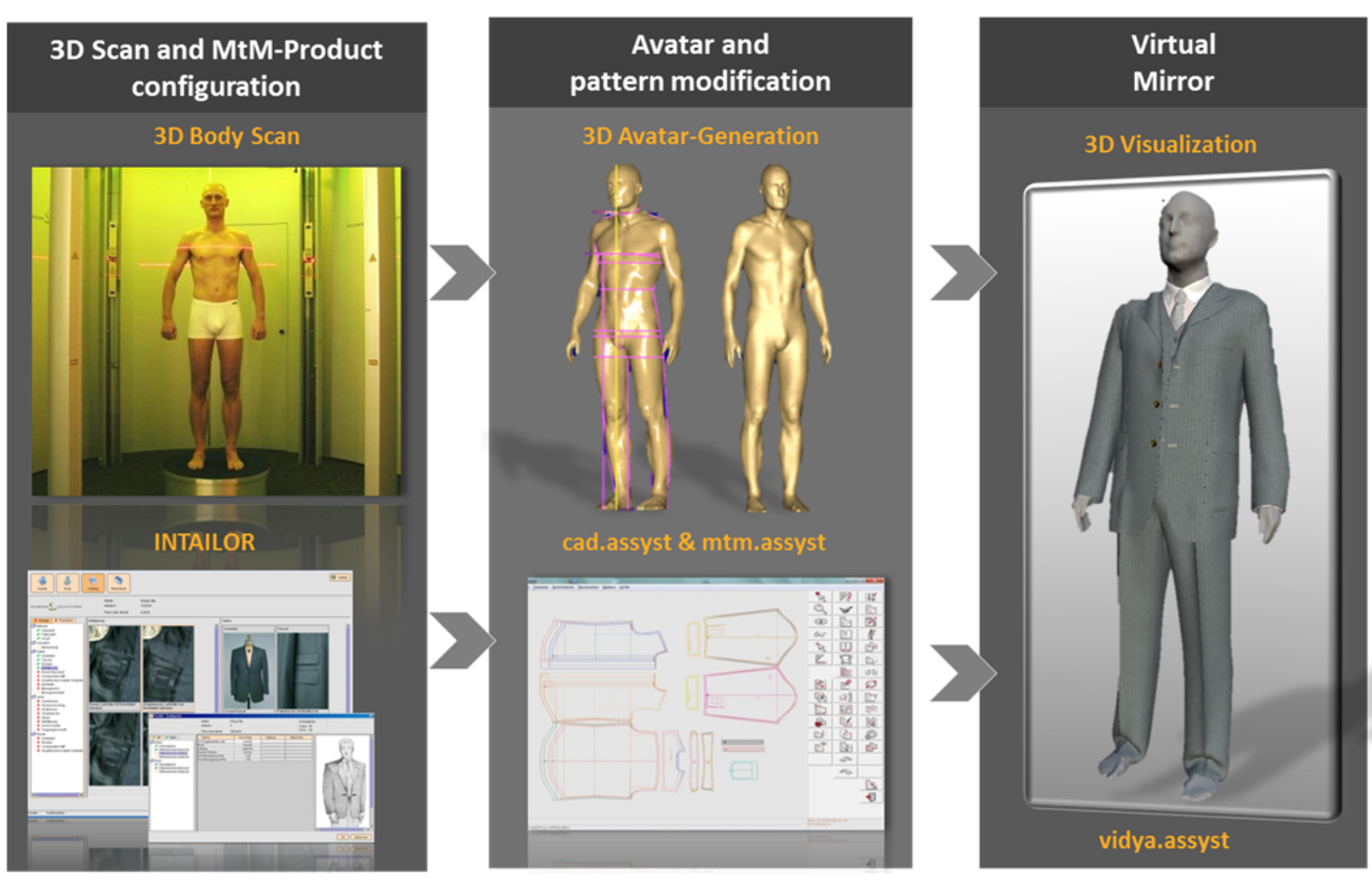

Fig. 1. Process overview.

The process chain described above can be realized with the INTAILOR infrastructure shown in Fig. 2. A set of applications and modules involved will be described below within the next chapters.

\subsection{Shop site}

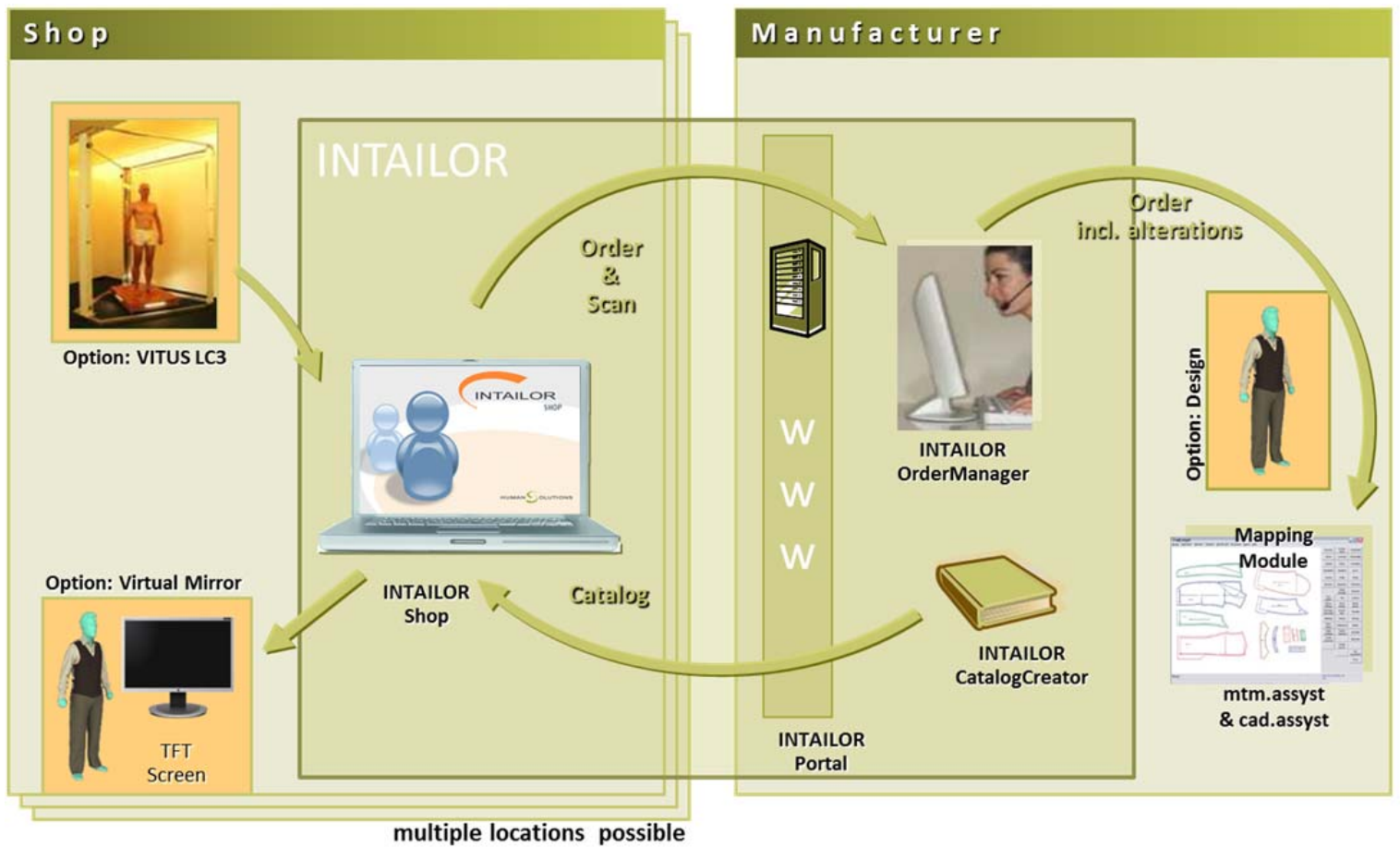

Fig. 2. Structure chart of INTAILOR. 


\subsubsection{INTAILOR Shop}

INTAILOR Shop features are:

- Catalog-based apparel item visualization

- Catalog-based apparel item configurator for error free design and MtM individualization

- Multiple catalogs possibility, for e.g. various brands, special catalogs or time-limited marketing activities

- Customer 3-D scan management

- Customer data management including historic order tracking

- Order copy function for quick configuration of repeat orders

- Complex shop calculation tool based on catalog variant selection including VAT handling and down payment management

- Activation of 3-D body scanner and assignment of scans to individual customers

- Electronic order creation with state of the art order XML data compilation and order state management

- Application administration and reports

- Freely configurable CRM tool of costumers profile (allowing combo boxes, $\mathrm{Y} / \mathrm{N}$ radio buttons, $\mathrm{Y} / \mathrm{N} / \mathrm{NC}$ checkboxes, text, text areas, numbers and dates)

- Enforced automatic upload of current catalogs and availabilities

INTAILOR Shop is installed on the scanner controlling computer using standard Windows Workstation OS systems.

The users should preferably at least once per day have the possibility to connect via Internet to the INTAILOR server (s. below) for the necessary data transfer and update needs. Standalone operation is possible, e.g. when traveling with laptop; orders will be sent again as soon as there is internet connection.

The INTAILOR Shop software is an indispensable prerequisite in order to drive and evaluate 3-D body scans.

\subsubsection{VITUS LC3}

The 3D BodyScanner VITUS LC3 features:

- Contact-free 3D body acquisition in order to gain garment measurements for the MtM process

- 3-column laser scanner with single triangulation creates a "digital twin" of the customer by taking around 200,000 surface points.

- The very small foot print of around $2.98 \mathrm{~m}^{2}$ and a height of $2.75 \mathrm{~m}$ allows an easy integration of the scanner into modern shop designs. The 3D BodyScanner is a new thrilling shopping experience.

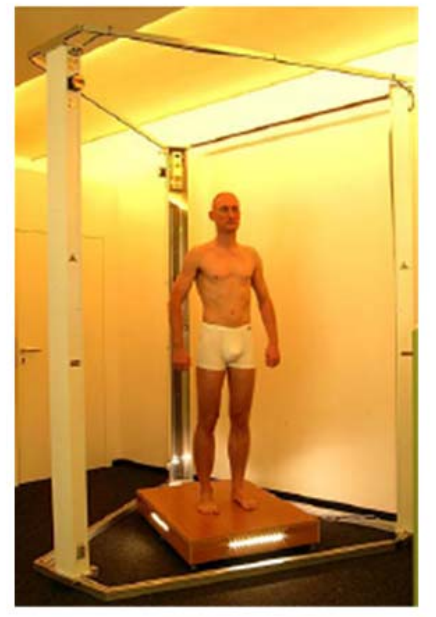

Fig. 3. VITUS LC 3.

\subsubsection{Virtual Mirror}

The Virtual Mirror is an integrated real time simulation of garments connected to INTAILOR. The simulation reflects selections in design and fitting. There is a possibility to do the selection of fabric, lining, buttons, etc. alongside with the adjustments done on variants and options of the product design. Base size and alteration values are related to the fitting.

Virtual Mirror is designed for the POS environment as well as for the back office together with the process without Try-On-Samples.
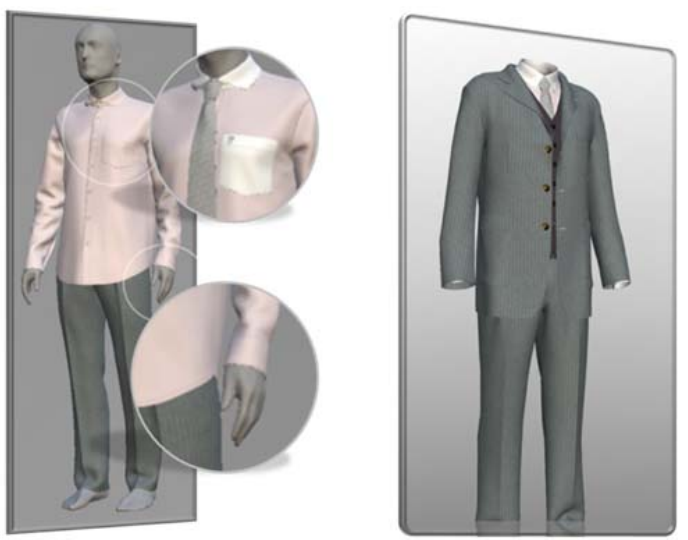

Fig. 4. Individual simulation of garments. 


\subsection{Manufacturer's site}

\subsubsection{INTAILOR Portal}

The INTAILOR Portal features:

- Web database connecting multiple INTAILOR Shop installations

- Automatic order administration accepting orders from shops and forwarding them to the according manufacturers

- Administrations of catalogs allowing their upload to the shops with the integrated validity check (manufacturer controls which catalog can be uploaded to which shop). Hosting of multiple catalogs in parallel by the server.

- Administration of availability of fabrics and options

The INTAILOR Portal is being installed at the manufacturer's site

\subsubsection{INTAILOR OrderManager}

The INTAILOR OrderManager features:

- Data set processing for commercial ERP systems and production systems, including automated pre-measurement of 3D body scans, if applicable

- Automated management of all current individual orders and (within a configurable spectrum) all past individual orders. All GUI presentation, filter and archiving tools necessary for individual order management

- Prepared for customer and case dependent software plug-ins for solution interface to all necessary ERP systems

\subsubsection{INTAILOR CatalogCreator}

As the MtM process constitutes a B2B2C relationship (business to business to customer) its reliability and efficiency highly depends on an error-free ordering process. All orders created by INTAILOR are based on the up-to-date catalogs created by the manufacturer. The INTAILOR CatalogCreator features:

- Set-up of consistency-tested catalogs with free design of variant visualizations, e.g. pictures

- Automatic transfer of components and price information from ERP systems

- Complex dependency configuration for individual components, also size/measurement concept-dependent; e.g. it is not possible to choose monogram as an option if no monogram text is defined

- Complex calculation module for sales price definition as basis for individual sales prices defined by the shop

- Ability to handle multiple catalogs, with copy functions, e.g. for target groups catalogs, special catalogs, sales activities

- Automatic upload procedure of catalogs via INTAILOR server to the shop software

\subsubsection{Design Option}

The Design Option can be described best as being an "electronic tailor". The Design Option is a fully automated solution for the complex process of converting customers' 3D body measurements to cutrelevant sizes and alteration values to the MtM modules of CAD systems. It includes a precise posture evaluation.

In detail it features:

- Software solution for automatic choice of most appropriate size from the graded size range, calculated directly from the customer's scan

- Definition of alteration rules and their limits

- Body measurements and finished measurements extraction

- Automatic differential measurement calculation

- Automatic posture evaluation and suggestion for alteration values 
- Data export file creation interfacing with the MtM tools of featured CAD systems, e.g. LECTRA, Gerber, assyst, etc.

The design option supports the process where no Try-On-Samples are necessary and the realization and calculation of the cut-relevant measurements happens at the manufacturer's site.

\subsection{Process chain}

\subsubsection{Collect customer data}

Customers preferring to buy individual garments typically have a long term partnership with their retailers. Within INTAILOR Shop (see chapter 0 ) the retailer sets up a customer database containing personal data, scan and order history

The scan wizard supports the retailer in taking individual scans of the customer. Today two scans in different postures are taken. There is one scan in standard standing posture for automatic measurement extraction and one scan in relaxed posture for virtual simulation of garments.

3D body scans taken with the VITUS LC3 (see chapter 2.2.2) produce a point cloud of the surface from the customer. To simulate garments on individual scans the point cloud has to be transformed to a mesh structure. With INTAILOR and its wizards the point cloud can be transformed to a mesh that can be used directly for virtual simulation and visualization.
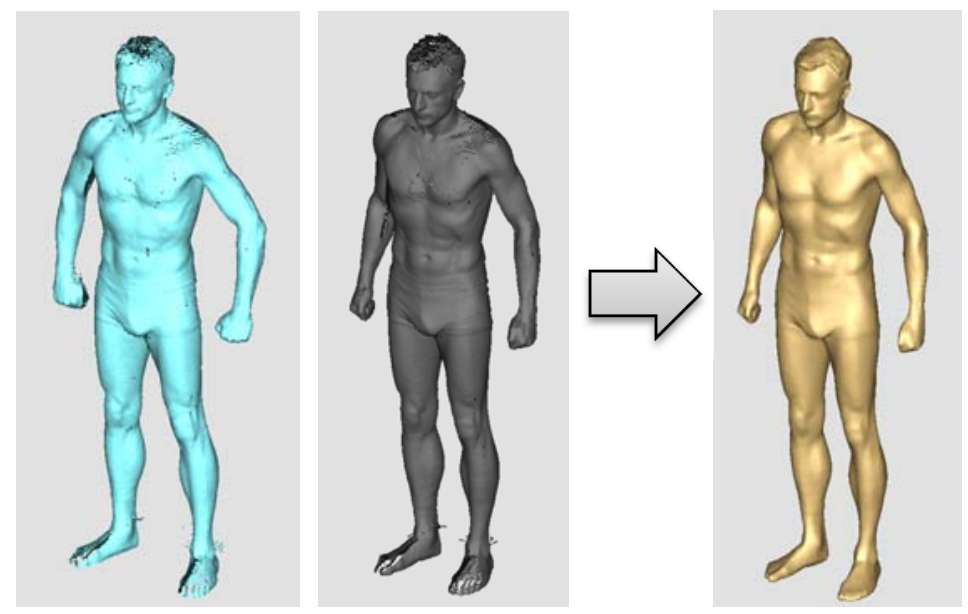

Fig. 5. Scan poses (left) automated measurement extraction, (center) garment simulation, (right) individual scanatar.

An avatar is the graphical representation of a person. It may take either a three- or a two-dimensional form. In the applications of the apparel industry an avatar is considered to be the representation of a complete individual person. 3D avatars are typically generated based on body measurements within an application to calculate a 3D model.

If the base of avatars changes from measurements to $3 \mathrm{D}$ body scans they are named scanatars. There are several algorithms available to generate scanatars.

\subsubsection{Product configuration and simulation}

The digital catalogs of products are being created by the manufacturer in INTAILOR CatalogCreator (see chapter 2.3.3) and are provided directly to POS. Within INTAILOR Shop various product catalogs are available for ordering products such as shirts, suits, etc.. Products can be configured in variants and options, materials including fabric and lining plus base size with alteration values. 


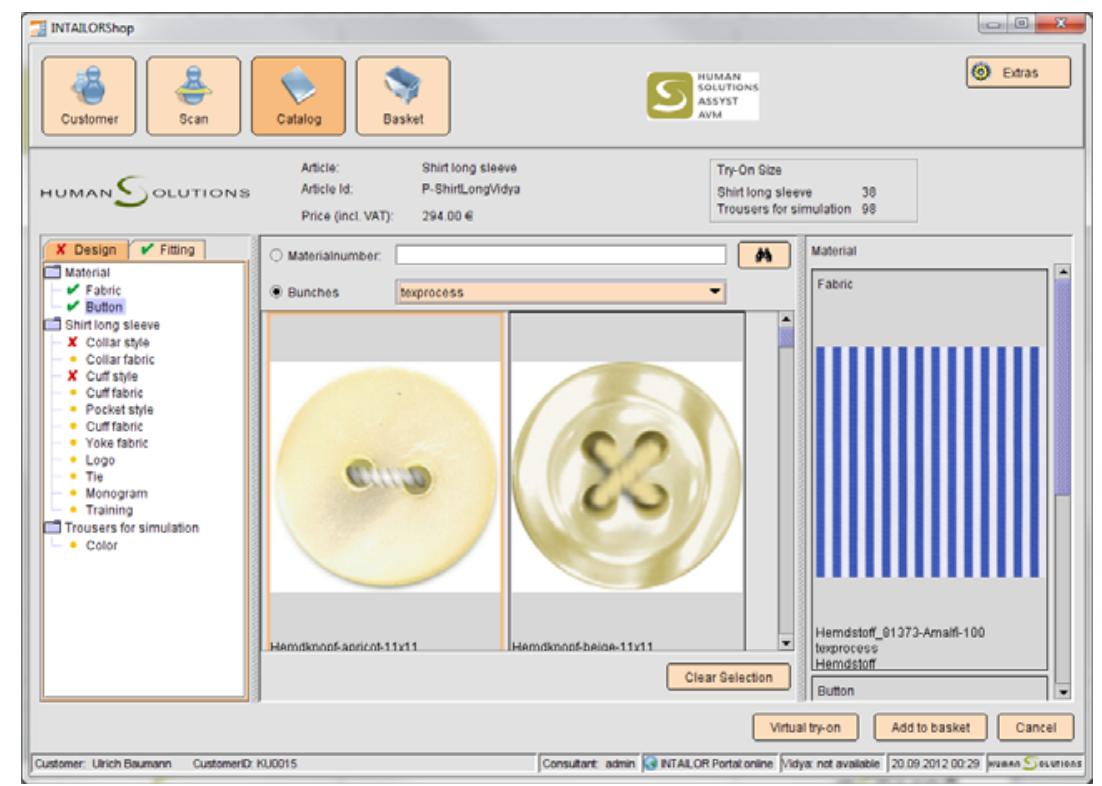

Fig. 6. Product configuration in INTAILOR Shop.

Once all obligatory variants and all obligatory measurements are selected the product can be sent to the Virtual Mirror and get simulated. Based on the individual scanatar of the customer the simulation of the individually configured garments is generated. Individually configured garment means individualization in design and fitting. Within the design changes regarding material and variants can be applied. Within the fitting changes regarding base size and alterations can be applied. Fig. 7 shows an individual scanatar with a shirt simulation. From left to right:

- normal fit

- left arm too short

- chest circumference too small

- normal fit in different fabric variants

(a)

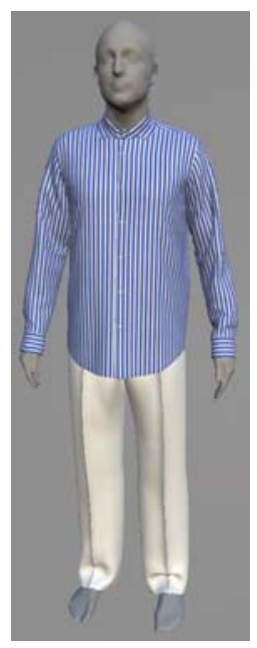

(b)

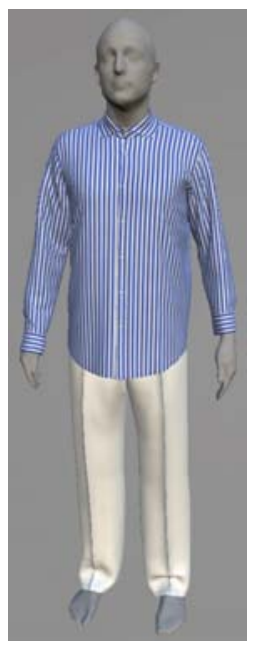

(c)

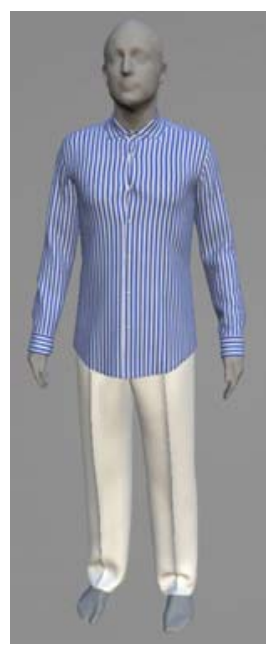

(d)

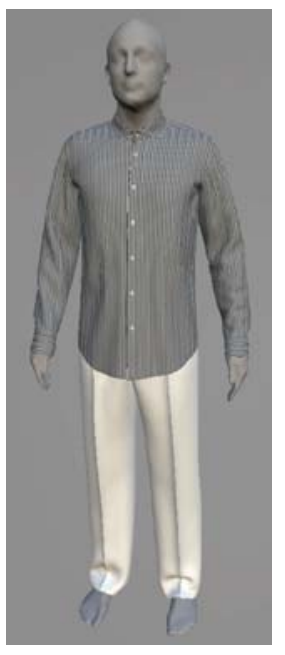

Fig. 7. Shirt simulation (a) normal fit, (b) left sleeve too short, (c) chest circumference too small, (d) normal fit in different fabric variants.

\section{Additional Applications}

In the apparel industry the size chart table with its measurements is one of the main know-how tools of a manufacturer. Each manufacturer or label focuses on its individual group of customers. This group can be defined by several criteria such as gender, age range, figure type, size, etc.. The certain group of customers is covered by a size range in the manufacturer's size chart. 


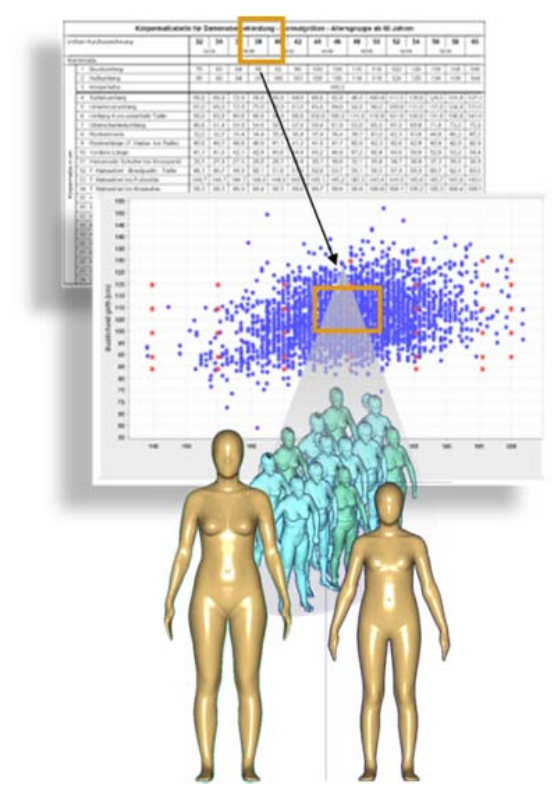

Fig. 8. Selection of a population.

Based on those statistical scanatars, physical mannequins can be produced. Depending on the garment different attributes of the physical mannequins are required Based on the results of the German size survey physical mannequins can be already ordered.
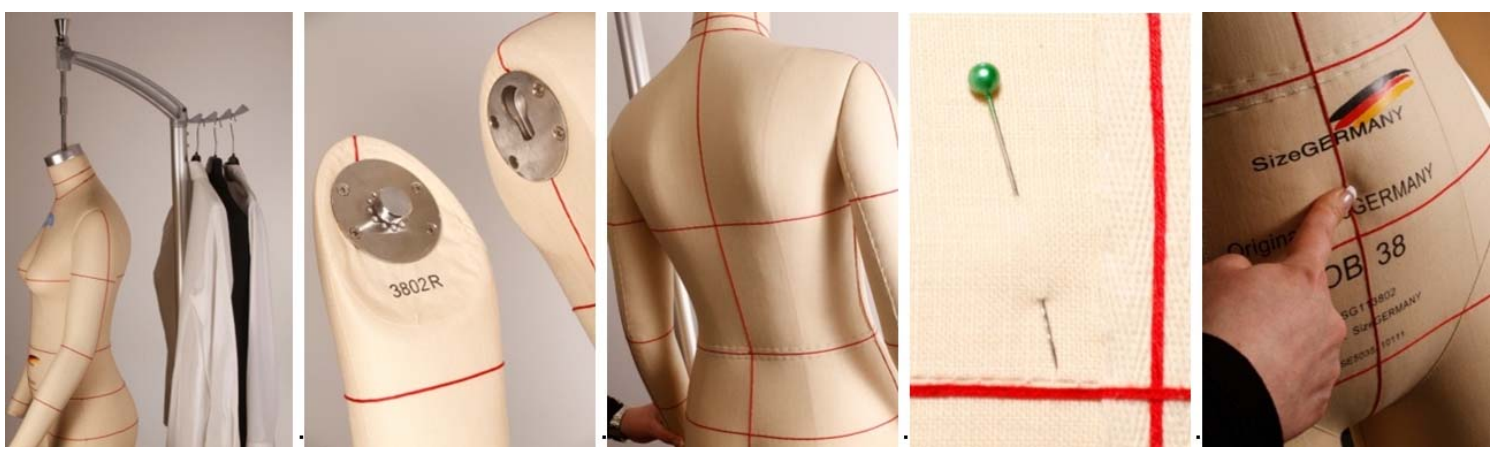

Fig. 9. Physical mannequins based on the German size Survey.

Beyond the simulation on individual scanatars the simulation on statistical scanatars with ready-towear garments can be used for representation in product configurations, web presentations and shop design.

To present virtual garments and products in print and web powerful rendering engines are strictly necessary. Such services are provided by $3^{\text {rd }}$ parties like RTT [3].

\section{Conclusions}

The range of applications using information based on the 3D scanatars is very wide. Today, the generation of scanatars is already available and widely used with high development potential in the future. Next step will be the generation of high-quality meshes acquired fully automatically without user interaction and within a short period of time. Thanks to the availability of individual scanatars such applications as Virtual Mirror can be used in shop environment to support the customer as well as the retailer. The future in this field will focus on the possibility of garment simulation with animated scanatars.

\section{References}

[1] INTAILOR: http://www.human-solutions.com/fashion/front content.php?idcat=142\&lang=7

[2] Virtual Mirror: http://www.human-solutions.com/fashion/front content.php?idcat=137\&lang=7

[3] RTT: www.rtt.ag

[4] Matthias Horx, Die Macht der Megatrends: http://www.horx.com/Reden/Macht-derMegatrends.aspx 